\title{
Lattice distortion of quantum cryocrystals under pressure
}

\author{
S.M. Tretyak, T.N. Antsygina, and Yu.A. Freiman \\ B. Verkin Institute for Low Temperature Physics and Engineering of the National Academy \\ of Sciences of Ukraine, 47 Lenin Ave., Kharkov 61103, Ukraine \\ E-mail: freiman@ilt.kharkov.ua
}

Received July 19, 2006

\begin{abstract}
The hcp lattice distortion parameter $\delta$, the deviation of $c / a$ ratio from the ideal hcp value $\sqrt{8 / 3}$, have been calculated for solid He under pressure taking into account two- and three-body interatomic forces. The resulting lattice distortion parameter is small and negative, that is the lattice is slightly flattened compared with the ideal hcp lattice. It monotonically increases in absolute value with pressure and reaches $10^{-3}$ for molar volume of $\sim 2.5 \mathrm{~cm}^{3} / \mathrm{mol}$. Such small distrotions are most likely outside of possibilities of x-ray or neutron experiments but can be detected by optical methods based on measurements of the birefringence. The data on $\delta$ can be used as a probe of the many-body forces.
\end{abstract}

PACS: 64.60.Cn, 67.80.-s, 67.90.+z, 81.40.Vw

Keywords: quantum cryocrystals, rare gas solids, lattice distortion parameter.

\section{Introduction}

As known, the structure of molecular crystals is governed by the close packing principle [1]. In the case of cryocrystals, the shape of the constituent entities - atoms in the case of rare gas solids ( $\mathrm{He}, \mathrm{Ne}, \mathrm{Ar}$, $\mathrm{Kr}, \mathrm{Xe})$ or diatomics in the case of the simplest molecular solids $\left(\mathrm{N}_{2}, \mathrm{CO}\right)$ is spherical or nearly spherical. The most close packing of hard spheres are either fcc or hcp. Among cryocrystals, only quantum atomic crystal - solid helium - and quantum molecular crystals $-J$-even solid hydrogens $\left(p-\mathrm{H}_{2}, o-\mathrm{D}_{2}\right.$ and $\left.\mathrm{HD}\right)-$ crystallize in hcp lattice whereas the heavier or classical cryocrystals both atomic ( $\mathrm{Ne}, \mathrm{Ar}, \mathrm{Kr}, \mathrm{Xe})$ and some diatomics $\left(\mathrm{N}_{2}, \mathrm{CO}\right)$ crystallize into cubic lattice.

Compared with the fcc lattice, the hcp lattice has an additional «degree of freedom» and is characterized by a certain $c / a$ ratio. In the case of close packing of hard spheres $c / a=\sqrt{8 / 3}$ (the «ideal» $c / a$ ratio or «ideal» hcp lattice). In the case of crystals with $c / a$ different from $\sqrt{8 / 3}$, the lattice have exactly the same symmetry as the ideal hcp lattice, i.e., the space groups of these crystals are identical for all values of $c / a$. The quantity

$$
\delta=c / a-\sqrt{8 / 3}
$$

the deviation of $c / a$ from the ideal hcp value, determines the second-order contribution to the crystal field [2]. Inspite of small amplitude, the presence of this harmonic is an important characteristic of hcp solids which distinguishes it from the ideal hep solids. As shown below, data on the deviation of $c / a$ from the ideal hcp value can be used to extract information about many-body interaction. The aim of this paper is to find theoretically the pressure dependence of the parameter $\delta$ and estimate the many-body contribution to the intermolecular potential for solid He. Some of the problems rised in this paper were discussed in short in Ref. 3.

Experimentally, no conclusive data exist about the value of the parameter $\delta$ at different pressures and temperatures. Vos et al. [4] using an optical method based on the presence of birefrigence in the hcp phase found that the deviation of $c / a$ from the ideal value is about -0.001 at the lowest density $\left(20.6 \mathrm{~cm}^{3} / \mathrm{mol}\right)$ and decreases in magnitude at higher densities $\left(-6 \cdot 10^{-4}\right.$ at $\left.1 \mathrm{kbar}\left(20.6 \mathrm{~cm}^{3} / \mathrm{mol}\right)\right)$. Neutron diffraction gave the value $c / a=1.63 \pm 0.03$ for a molar volume of $18.51 \mathrm{~cm}^{3} / \mathrm{mol}$ [5], but a recalculation of Henshaw's data by Donohue [6] resulted in the value $c / a=1.612 \pm 0.004$. X-ray diffraction gave $c / a=$ $=1.628 \pm 0.009$ at a molar volume $20.66 \mathrm{~cm}^{3} / \mathrm{mol}$ [7] and $c / a=1.627 \mathrm{~cm}^{3} / \mathrm{mol}$ at $17.4 \mathrm{~cm}^{3} / \mathrm{mol}$ [8]. X-ray 
studies of helium crystals at high pressures gave the value $1.630 \pm 0.005[9-11]$. These studies did not reveal a systematic changing in $c / a$ with pressure though it should be noted, that the uncertainty in determination of $c / a$ is too high and leave a room for manifesting the effect.

In the case of solid hydrogens, at zero pressure $\delta$ is of the same order of magnitude as in solid helium $[2,12]$. The effect increases with pressure and at pressures about $100 \mathrm{GPa}$ the observed deviation of $c / a$ ratio from ideal value is as high as $-0.05[13,14]$. Using the same approach for $\mathrm{H}_{2}$ that was developed for solid He we calculated the contribution of the isotropic part of the intermolecular potential to $\delta$ and found that it is negligible compared with the observed value. This points to the significance of the rotational degrees of freedom of hydrogen molecules. The theory that takes into account the rotational part of the Hamiltonian will be given elsewhere.

In view of the fact that the term CRYOCRYSTALS has been put in into circulation by Antonina Fedorovna Prikhot'ko, this paper may perhaps be an appropriative contribution to an issue of Fizika Nizkikh Temperatur (Low Temperature Physics) dedicated to her centenary.

\section{Solid helium}

At small pressures the interparticle interaction in helium is described by Aziz potential [15]

$$
U_{p}(R)=\exp (-\alpha-\beta R)-f_{c}(R) \sum_{n=6,8,10} C_{n} R^{-n} .
$$

Here $R$ is the interparticle distance, $\alpha=2.925, \quad \beta=$ $=2.381405$ a.u., coefficients $C_{n}$ are equal to (in atomic units) $C_{6}=1.45995, C_{8}=14.2195, C_{10}=$ $=187.1926$.

The damping fuction

$$
f_{c}(R)= \begin{cases}\exp \left[-\left(R^{*} / R-1\right)^{2}\right], & R<R^{*} \\ 1, & R>R^{*}\end{cases}
$$

where $R^{*}=1.28 R_{m}, R_{m}=5.4379$ a.u. is the position of the potential minimum.

However, the pair potential does not describe properly the properties of the system under very high pressure, and many-body effects should be taken into account. Here we restrict ourselves to the three-particle interaction, which consists of three-body dispertion forces (Axilrod-Teller forces) and tree-body exchange forces.

Let three paticles form a triangular with sides $r_{1}$, $r_{2}, r_{3}$ and angles $\varphi_{1}, \varphi_{2}, \varphi_{3}$. Then the total three-body potential $U_{\text {tr }}$ is given by

$$
\begin{aligned}
U_{\mathrm{tr}}=\{ & \left.-A \exp \left[-\alpha\left(r_{1}+r_{2}+r_{3}\right)\right]+\frac{C_{\mathrm{tr}}}{r_{1}^{3} r_{2}^{3} r_{3}^{3}}\right\} \times \\
& \times\left(1+3 \cos \varphi_{1} \cos \varphi_{2} \cos \varphi_{3}\right),
\end{aligned}
$$

where the coefficients (in a.u.) are $A=30.644, \alpha=$ $=1.0245, C_{\mathrm{tr}}=1.4807$ [16].

Due to the quantum nature of solid helium, the effect of zero-point oscillations is rather noticable when the pressure dependence of the crystal structure is investigated, although it will be shown below that the role of zero-point oscillations reduces considerably as pressure increases. At the same time, the thermal phonon contribution can be neglected. Even at room temperatures (typical temperatures of the most of high-pressure experiments) the thermal phonon contribution is small compared with that of zero-point oscillations. The ratio of these contributions to the equation of state can be easily estimated in the Debye approximation to give

$$
P_{\text {th }} / P_{\text {z.p. }} \sim\left(T / \Theta_{D}\right)^{4} .
$$

The Debye temperature $\Theta_{D}$ increases very rapidly with pressure [11] and the condition $\left(T / \Theta_{D}\right)^{4}<<1$ is fulfilled for the whole considered range of pressures.

As a result, the Hamiltonian of the system takes the form [3]

$$
H=U_{p}+U_{\mathrm{tr}}+U_{\text {z.p. }},
$$

where $U_{\text {z.p. }}$ is the energy of zero-point oscillations.

In the following, the pressure region where zero-point effects play a decisive role will be excluded from consideration, so in calculations of the zero-point contribution standart methods of lattice dynamics have been used, in particular, the Einstein approximation. In this approximation the zero-point energy can be found in the following way:

$$
U_{\text {z.p. }}=\frac{\hbar}{2} \sum_{\alpha} \omega_{\alpha}
$$

where $\omega_{\alpha}(\alpha=x, y, z)$ are frequences of the harmonic oscillations of helium atoms near their equilibrium positions.

Let us use the fact that the parameter $\delta$ is small $(\delta<<1)$ and expand the free energy of the system in powers of $\delta$ restricting ourselves to the terms of the second order in $\delta$ :

$$
F(\delta)=F_{0}+b_{1} \delta+b_{2} \delta^{2},
$$

where $F_{0}$ is the free energy of the ideal hcp lattice, $b_{i}$ $(i=1,2)$ are the coefficients depending on the $\mathrm{pa}^{-}$ rameters of the system. 
Minimizing $F(\delta)$ over $\delta$, we obtain

$$
\delta=-\frac{b_{1}}{2 b_{2}} .
$$

Due to the short-range character of the interparticle interaction in helium [17] it may seem that consideration only a few nearest shells of neighbors spheres is quite enough for calculations. It is really the case for $b_{2}$, the coefficient at the quadratic term in $\delta$. For this term the first coordination sphere alone make a main positive contribution, and contributions from more distant spheres tend rapidly to zero. However, the linear in $\delta$ contributions to the free energy from the first two shells of neighbors exactly equal to zero $\left(b_{1}=0\right)$. It should be noted that the exact vanishing of the contributions from the first two shells in the hcp lattice is not due to the symmetry. Moreover, the contributions to $b_{1}$ from nearest and next nearest neighbors vanish owing to different reasons. In the case of the first shell, the contribution from the nearest neighbors (nn) in the basic plane cancels the contribution from the nearest neighbors below and above the basic plane. Such cancellation takes place due to the accidental equality of all the nn intermolecular separations when $c / a=\sqrt{8 / 3}$. For the next nearest neighbors the contributions to $b_{1}$ vanish individually from each of the six molecules. The contributions from more distant shells of neighbors decrease rather slowly and tend to alternate in signs, so to find the pressure dependence of $\delta(P)$ we have to consider a large number of shells.

The contribution to $b_{1}$ from the third coordination sphere containing only two molecules at the distance $R=\sqrt{8 / 3}$ from the central molecule leads to $b_{1}>0$ resulting in $\delta<0$. Thus, in this approximation as the pressure increases the flattened hcp structure with the ratio $c / a<\sqrt{8 / 3}$ is more favorable. However, the interaction with each neighbor from the fourth coordination sphere makes a positive contribution to $b_{1}$. Since the fourth sphere contains 18 neighbors its total contribution to $b_{1}$ substantially exceeds the contribution from the third coordination sphere. As a result, the contribution from these two spheres leads to positive $\delta$.

Theoretically obtained dependences of the parameter $\delta$ on the specific volume for solid ${ }^{4} \mathrm{He}$ are shown in Fig. 1. Solid curves were obtained for both pair and three-body interactions and dashed curves were calculated for the pair interaction only. These curves were calculated with allowance for zero-point oscillations. The curves are numbered in accodance with quantities of the coordination spheres that have been taken into account. The Figure reflects the above-mentioned strong dependence of the calculated results on the number of the coordination spheres. As it is seen, the

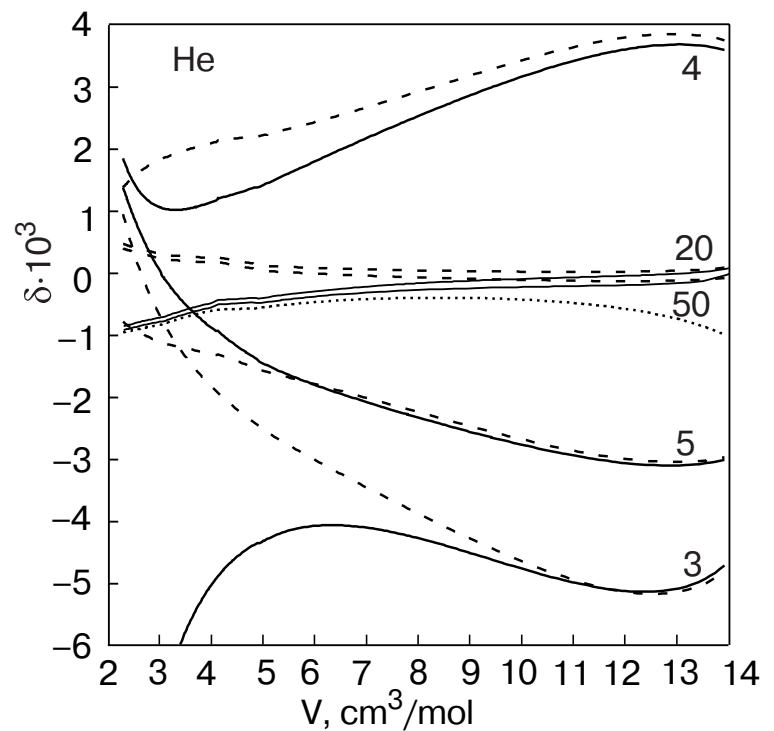

Fig. 1. Lattice distortion parameter $\delta$ for solid $\mathrm{He}$ as a function of molar volume calculated: with the pair plus three-body potential (solid curves); with the pair Aziz potential (dashed curve). The numbers at curves show how many shells of neighbors was taken into account in the calculations. The dotted curve (corresponding to 50 shells) was calculated without regard for zero-point oscillations.

convergence of $\delta$ to its limiting value is slow. To obtain unumbiguous quntitative results it is necessary to take into account rather distant shells. This situation is not unusual for the systems with long-range interaction, such as ionic crystals and the systems in which the dipole-dipole interaction plays the main role. But such situation for the rare gas solids with short-range interaction as far as we know is met for the first time.

When the number of the shells increases, the quantity $\delta$ tends to its limiting value, which depends on the specific volume. Increase in the number of shells over $\sim 20$ ceases to influence the calculated results uppreciably as is demonstrated by a small difference between the curves for 20 and 50 shells. As can be seen, resulting $\delta$ is negative and increases in magnitude with decreasing specific volume monotonically. At the molar volume $2.5 \mathrm{~cm}^{3} /$ mol (approximately ten-fold compression, $P \sim 60 \mathrm{GPa}) \delta \approx-0.001$.

The role of the many-body forces can be seen from the comparison of solid curves which were obtained with allowance for pair and three-body interactions and dashed curves which were calculated for the pair interaction only. It is important that the effect not only increases with pressure but it leads to a qualitative difference in the behavior of $\delta(V)$. Namely, the action of the pair forces results in positive $\delta$ (the elongation of the hexagonal lattice) whereas the potential that includes the three-body forces tends to flatten the lattice. 


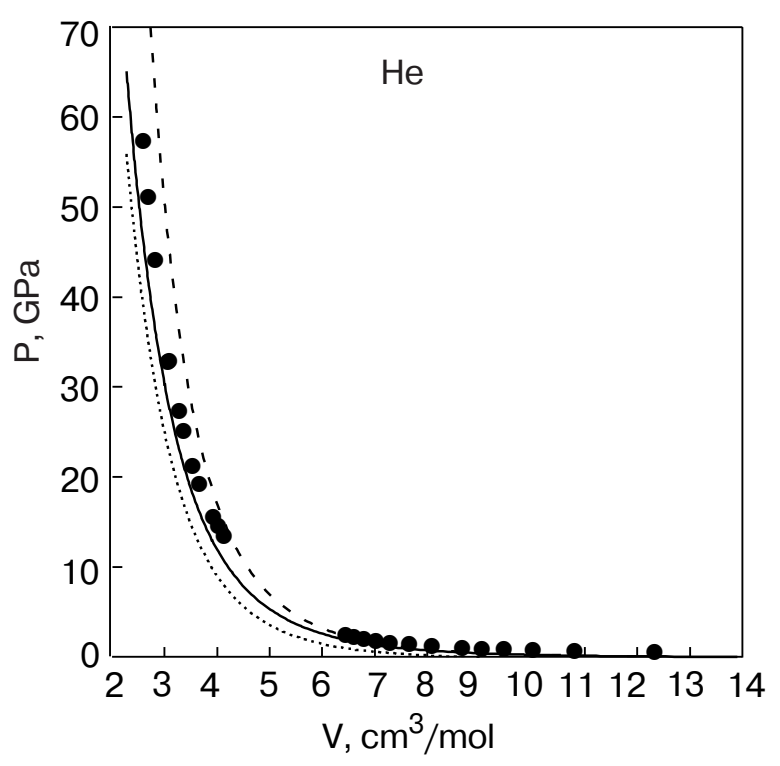

Fig. 2. The calculated $T=0 \mathrm{~K}$ equation of state for solid He. Solid symbols are experimental data from Refs. 9, 10 and 18. Solid, dashed and dotted curves refer to the same conditions as in Fig. 1 corrected to $T=0 \mathrm{~K}$.

The dotted curve (corresponding to 50 shells) was calculated without regard for zero-point oscillations. It is seen, first, that zero-point oscillations is a factor favoring the ideal hcp structure, and second, that the contribution of zero-point oscillations decreases markedly with rising pressure.

Let us now turn to the equation of state $P(V)$ for ${ }^{4} \mathrm{He}$. The deviation from the ideal structure very slightly affects the behavior of $P(V)$ so that for its calculation it is enough to retain only first term in Eq. (7). The calculated dependences of $P(V)$ are shown in Fig. 2 along with the experimental results [9]. It is seen that the pair potential is too stiff and overestimates the pressure values calculated with its help considerably. The contribution from the three-body interaction increases rapidly with pressure and at $P \geq 10 \mathrm{GPa}$ the pair interaction approximation becomes inadequate. The contribution from zero-point oscillations to the pressure is noticible in the whole region where the measurement of $P(V)$ were made although its role is more pronounced at low $P$.

\section{Solid hydrogen}

Similar calculations were performed for the isotropic part of the intermolecular potential for solid hydrogen. No account has been taken in these calculations of the rotational part of the Hamiltonian. It has been shown that the contribution from the isotropic part of the intermolecular potential to the distortion parameter $\delta$ is a small part of the total effect implying that the main effect is due to the rotational degrees of

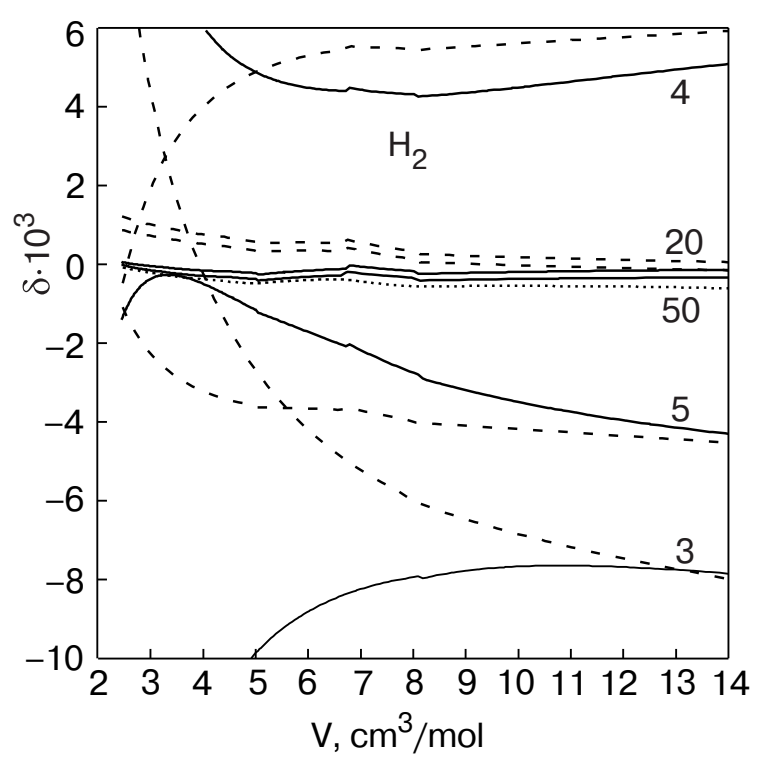

Fig. 3. The contribution to the lattice distortion parameter $\delta$ from the isotropic part of the intermolecular potential for solid $p-\mathrm{H}_{2}$ as a function of molar volume. Solid, dashed and dotted curves refer to the same conditions as in Fig. 1.

freedom of hydrogen molecules. Results of these calculations are given in Fig. 3. As in Fig. 1, the curves are numbered in accordance with the quantities of shells which were taken into account; the solid curves are referred to the total Hamiltonian (5), dashed curves are results when the many-body interaction was disregarded, and the dotted curve was obtained when zero-point oscillations were omitted (for 50 shells). Qualitatively, these results are similar to that obtained for solid He: when summing up the contributions from progressively distant shells of neighbors the convergency is rather slow and the partial contributions tend to alternate in signes; the lattice distortion parameter $\delta$ resulting from the pair potential and that from many-body forces have different signs and near compensate each other.

\section{Conclusions}

Comparing two close-packed lattices, fcc and hcp, the latter has an additional structure characteristics: the lattice distortion parameter $\delta$ - the deviation of $c / a$ ratio from the ideal hcp value $\sqrt{8 / 3}$. In this paper the pressure dependence of this parameter have been calculated for solid He. We used the intermolecular potential which was represented by a sum of the pair Aziz potential and three-body potential consisting of the Axilrod-Teller dispersive forces and the three-body exchange interaction. Calculations of the pressure-volume relation revealed that the Aziz pair potential is too stiff and give rise to overastimated values of pressure. This drawback of the pair Aziz potential is 
compensated by the negative contribution from the three-body potential. The lattice distortion parameter $\delta$ resulting from the pair potential and that from many-body forces have different signs and tend to cancell each other. The resulting lattice distortion parameter is small and negative, that is the lattice is slightly flattened compared with the ideal hcp lattice. It monotonically increases in magnitude with pressure and reaches $10^{-3}$ for molar volume of $\sim 2.5 \mathrm{~cm}^{3} / \mathrm{mol}$. Such small distortions are most likely outside of possibilities of x-ray or neutron experiments but can be detected by optical methods based on measurements of the birefringence. The data on $\delta$ can be used as a probe of the many-body forces.

1. A.I. Kitaigorodsky, Molecular Crystals, Academic Press, New York (1973).

2. J. van Kranendonk, Solid Hydrogen, Plenum Press, New York (1983).

3. Yu.A. Freiman, S.M. Tretyak, A. Jeżowski, and R.J. Hemley, J. Low Temp. Phys. 126, 703 (2002); ibid. 122, 537 (2001).

4. J.E. Vos, R.V. Kingma, F.J. van der Gaag, and B.S. Blaisse, Phys. Lett. A24, 738 (1967).

5. D.G. Henshaw, Phys. Rev. 109, 328 (1958).

6. J. Donohue, Phys. Rev. 114, 1009 (1959).
7. R.L. Mills and A.F. Schuch, Proc. VIII Int. Conf. Low Temp. Physics, London (1962), p. 423.

8. A.F. Schuch and R.L. Mills, 6-th Int. Congr. of the Int. Union of Crystall., Rome (1963), p. A21.

9. H.K. Mao, R.J. Hemley, Y. Wu, A.P. Jephcoat, L.W. Finger, C.S. Zha, and W.A. Basset, Phys. Rev. Lett. 60, 2649 (1988).

10. P. Louybeyre, R. LeToullec, J.P. Pinceaux, H.K. Mao, J. Hu, and R.J. Hemley, Phys. Rev. Lett. 71, 2272 (1993).

11. C.S. Zha, H.K. Mao, and R.J. Hemley, Phys. Rev. B70, 174107 (2004).

12. H. Katsuki, M. Fushitani, and T. Momose, Fiz. Nizk. Temp. 29, 1093 (2003) [Low Temp. Phys. 29, 832 (2003)].

13. P. Louybeyre, R. LeToullec, D. Hausermann, M. Hanfland, R.J. Hemley, H.K. Mao, and L.W. Finger, Nature 383, 702 (1996).

14. H. Kawamura, Y. Akahama, S. Umemoto, K. Takemura, Y. Ohishi, and O. Shimomura, J. Phys.: Condens. Matter 14, 10407 (2002).

15. R.A. Aziz, V.P.S. Nain, J.S. Carley, W.L. Taylor, and G.T. McConville, J. Chem. Phys. 70, 4330 (1979).

16. P. Louybeyre, Phys. Rev. Lett. 58, 1857 (1987).

17. M.I. Klein and J.A. Venables, Rare Gas Solids, Academic, London (1976).

18. J.W. Stewart, Phys. Rev. 129, 1950 (1963). 